\title{
Supporting Information for: Vesicle Origami and the Influence of Cholesterol on Lipid Packing
}

Radu Tanasescu ${ }^{\dagger}$, Martin A. Lanz ${ }^{\dagger}$, Dennis Mueller ${ }^{\dagger}$, Stephanie Tassler ${ }^{\ddagger}$, Takashi Ishikawa ${ }^{\S}$, Renate Reiter $^{\top *}$, Gerald Brezesinski ${ }^{\dagger *}$, Andreas Zumbuehl ${ }^{\dagger *}$

$\dagger$ Department of Chemistry, University of Fribourg, Chemin du Musée 9, 1700 Fribourg, Switzerland

\$Max Planck Institute of Colloids and Interfaces, Science Park Potsdam-Golm, 14476 Potsdam, Germany

§Paul Scherrer Institute (PSI), OFLB/010, 5232 Villigen PSI, Switzerland

qUniversity of Freiburg, Experimental Polymer Physics, Hermann Herder Strasse 3, 79104 Freiburg, Germany

Freiburg Centre for Interactive Materials and Bioinspired Technologies (FIT), 79110

Freiburg, Germany

\section{Brewster-angle Microscopy}

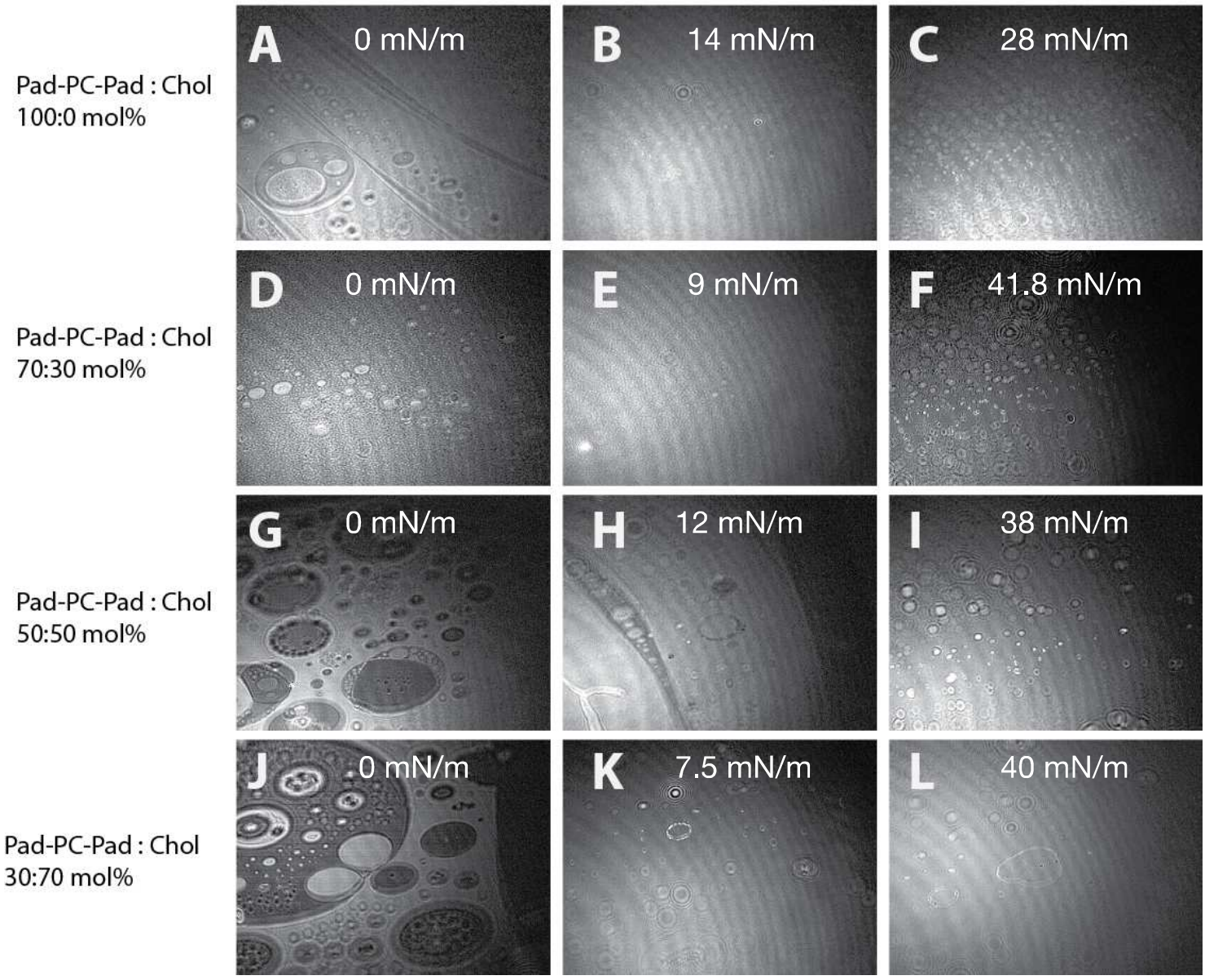

Figure S1. Brewster angle micrographs of Pad-PC-Pad:cholesterol monolayers at different surface pressures at room temperature. Each micrograph is scaled to $535 \mu \mathrm{m} \times 510 \mu \mathrm{m}$. 


\section{SAXS and WAXS Bilayer Data}

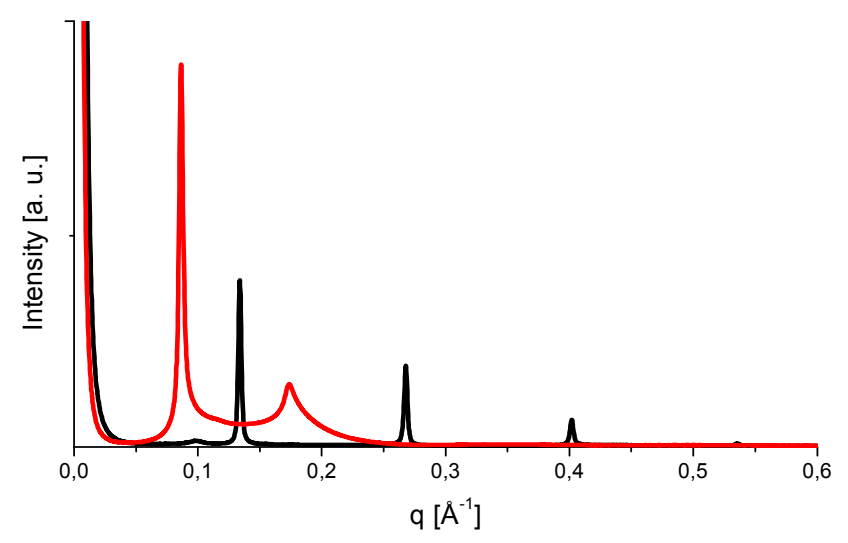

Figure S2: Small Angle X-Ray Scattering pattern of Pad-PC-Pad (20 wt $\%$ lipid dispersion in water, black line $-15^{\circ} \mathrm{C}$, red line $-40{ }^{\circ} \mathrm{C}$ ) showing the interdigitation below the main transition temperature $\mathrm{T}_{\mathrm{m}}=37^{\circ} \mathrm{C}$ and the absence of interdigitation above $\mathrm{T}_{\mathrm{m}}$.

Table TS1: Peak positions of Pad-PC-Pad in Small-Angle X-Ray Scattering.

\begin{tabular}{|l|l|l|l|l|l|l|}
\hline $\begin{array}{l}\text { Pad-PC- } \\
\text { Pad }\end{array}$ & $\mathbf{q}_{1}\left[\AA^{-1}\right]$ & $\mathbf{q}_{2}\left[\AA^{-1}\right]$ & $\mathbf{q}_{3}\left[\AA^{-1}\right]$ & $\mathbf{q}_{\mathbf{4}}\left[\AA^{-1}\right]$ & $\mathbf{q}_{1}{ }^{*}\left[\AA^{-1}\right]$ & $\mathbf{q}_{2}{ }^{*}\left[\AA^{-1}\right]$ \\
\hline $\begin{array}{l}\mathbf{1 5}{ }^{\circ} \mathbf{C} \\
\text { lamellar }\end{array}$ & 0.134 & 0.268 & 0.402 & 0.536 & & \\
\hline $\begin{array}{l}\mathbf{4 0}{ }^{\circ} \mathbf{C} \\
\text { lamellar }\end{array}$ & & & & & 0.087 & 0.175 \\
\hline
\end{tabular}




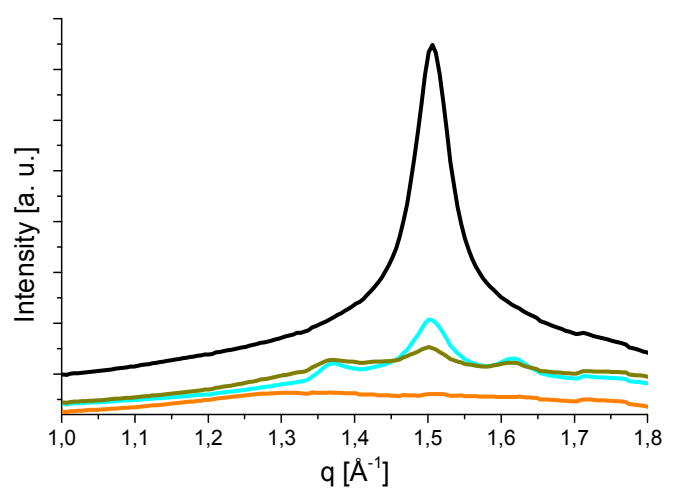

Figure S3: WAXS of $20 \mathrm{wt} \%$ Pad-PC-Pad dispersions in water at $15{ }^{\circ} \mathrm{C}$ (black - Pad-PCPad, cyan - Pad-PC-Pad with 10 mol\% cholesterol, dark yellow - Pad-PC-Pad with $20 \mathrm{~mol} \%$ cholesterol, orange - Pad-PC-Pad with 40 mol\% cholesterol).

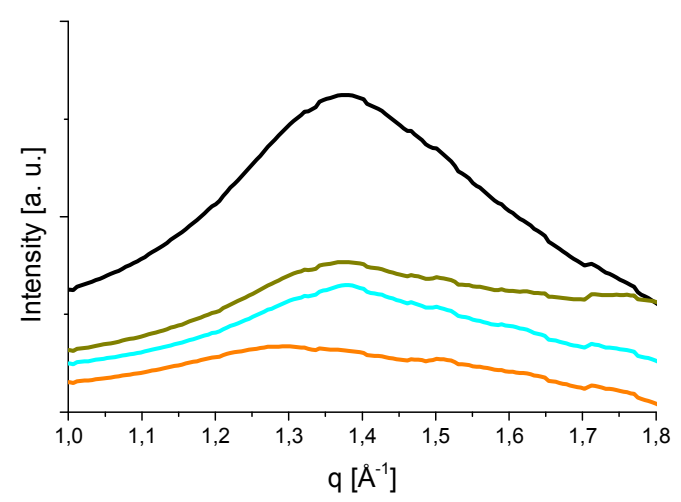

Figure S4: WAXS of $20 \mathrm{wt} \%$ Pad-PC-Pad dispersions in water at $40{ }^{\circ} \mathrm{C}$ (black - Pad-PCPad, cyan - Pad-PC-Pad with 10 mol\% cholesterol, dark yellow - Pad-PC-Pad with $20 \mathrm{~mol} \%$ cholesterol, orange - Pad-PC-Pad with $40 \mathrm{~mol} \%$ cholesterol). 
Table TS2: Peak positions in WAXS patterns and calculated cross-sectional areas per chain.

\begin{tabular}{|c|c|c|c|c|c|}
\hline sample & $\mathbf{T}\left[{ }^{\circ} \mathbf{C}\right]$ & $\mathbf{q}_{1 \mathrm{xy}}\left[\AA^{-1}\right]$ & $\mathbf{q}_{\mathbf{2 x y}}\left[\AA^{-1}\right]$ & $\mathbf{q}_{\mathbf{3 x y}}\left[\AA^{-1}\right]$ & $\mathbf{A}_{0}\left[\AA^{2}\right]$ \\
\hline \multirow[t]{2}{*}{ Pad-PC-Pad } & 15 & 1.506 & & & 20.1 \\
\hline & 40 & 1.38 halo & & & 23.9 \\
\hline \multirow{2}{*}{$\begin{array}{c}\text { Pad-PC-Pad } \\
10 \mathrm{~mol} \% \\
\text { Cholesterol }\end{array}$} & 15 & 1.377 & 1.504 & 1.615 & 20.6 \\
\hline & 40 & 1.37 halo & & & 24.3 \\
\hline \multirow[t]{2}{*}{$\begin{array}{c}\text { Pad-PC-Pad } \\
20 \mathrm{~mol} \% \\
\text { Cholesterol }\end{array}$} & 15 & 1.365 & 1.501 & 1.615 & 20.7 \\
\hline & 40 & 1.35 halo & & & 25.0 \\
\hline
\end{tabular}

